\title{
Positive Solutions for Class of State Dependent Boundary Value Problems with Fractional Order Differential Operators
}

\author{
Dongyuan Liu, Zigen Ouyang, and Huilan Wang \\ School of Mathematics and Physics, University of South China, Hengyang 421001, China \\ Correspondence should be addressed to Zigen Ouyang; zigenouyang@163.com
}

Received 13 April 2014; Revised 4 August 2014; Accepted 4 August 2014

Academic Editor: Ali H. Bhrawy

Copyright (C) 2015 Dongyuan Liu et al. This is an open access article distributed under the Creative Commons Attribution License, which permits unrestricted use, distribution, and reproduction in any medium, provided the original work is properly cited.

\begin{abstract}
We consider the following state dependent boundary-value problem $D_{0+}^{\alpha} y(t)-p D_{0+}^{\beta} g(t, y(\sigma(t)))+f(t, y(\tau(t)))=0,0<t<1$; $y(0)=0, \eta y(\sigma(1))=y(1)$, where $D^{\alpha}$ is the standard Riemann-Liouville fractional derivative of order $1<\alpha<2,0<\eta<1, p \leq 0$, $0<\beta<1, \beta+1-\alpha \geq 0$ the function $g$ is defined as $g(t, u):[0,1] \times[0, \infty) \rightarrow[0, \infty)$, and $g(0,0)=0$ the function $f$ is defined as $f(t, u):[0,1] \times[0, \infty) \rightarrow[0, \infty) \sigma(t), \tau(t)$ are continuous on $t$ and $0 \leq \sigma(t), \tau(t) \leq t$. Using Banach contraction mapping principle and Leray-Schauder continuation principle, we obtain some sufficient conditions for the existence and uniqueness of the positive solutions for the above fractional order differential equations, which extend some references.
\end{abstract}

\section{Introduction}

Fractional order differential equations has useful applications in many fields, such as physics, mechanics, chemistry, engineering, biology, and so on. There has been a significant development in fractional differential equations (e.g., [1-9]). In the previous papers, some authors investigated fractional order partial differential equations [10-15]. For example, $\mathrm{Wu}$ [15] used the wavelet operational method for solving fractional partial differential equations numerically. Since it is one of the important fields to be concerned with the boundary value problems for fractional order differential equations, some authors considered the existence of positive solutions for fractional differential equations or systems with boundary value conditions [16-25] and the stability [26].

As early as 1994, Delbosco [27] investigated the nonlinear Dirichlet-type problem

$$
\begin{aligned}
x^{\alpha-1}\left(D_{0+}^{\alpha} y\right)(x) & =f(y(x)), \quad 0<x<1,1<\alpha<2, \\
y(0) & =y^{\prime}(1)=0,
\end{aligned}
$$

where $D^{\alpha}$ is $\alpha$ order Riemann-Liouville derivative. The author had proved that if $f$ is a Lipschitz function, then the problem has at least one solution $y(x)$ in a certain subspace of $C[0,1]$ in which the fractional derivative has a Hölder property.

Later, using some fixed point theorems, Bai and Lü [20] obtained the existence of positive solutions of the following equation with boundary value conditions

$$
\begin{aligned}
& D_{0+}^{\alpha} y(t)+f(t, y(t))=0, \quad 0<t<1,1<\alpha<2, \\
& \quad y(0)=y(1)=0, \\
& \quad \text { or } \quad y(0)+y^{\prime}(0)=y(1)+y^{\prime}(1)=0 .
\end{aligned}
$$

More recently, Bai [9] also considered the following boundary value problem

$$
\begin{aligned}
D_{0+}^{\alpha} y(t)+f(t, y(t)) & =0, \quad 0<t<1,1<\alpha \leq 2, \\
y(0) & =0 \\
\beta y(\eta) & =y(1) .
\end{aligned}
$$

By constructing a Green's function, and using contraction map principle, the author obtained some existence conditions of positive solutions for (3). 
Motivated by the above references, we consider a state dependent boundary value problem with fractional order differential operators

$$
\begin{gathered}
D_{0+}^{\alpha} y(t)-p D_{0+}^{\beta} g(t, y(\sigma(t)))+f(t, y(\tau(t)))=0, \\
0<t<1, \\
y(0)=0, \\
\eta y(\sigma(1))=y(1),
\end{gathered}
$$

where $D^{\alpha}$ is the standard Riemann-Liouville fractional derivative of order $1<\alpha<2,0<\beta<1,0<\eta<1, p \leq 0$, $\beta+1-\alpha \geq 0,1-\eta \sigma^{\alpha-1}(1)>0$; the function $g$ is defined as $g(t, u):[0,1] \times[0, \infty) \rightarrow[0, \infty)$, and $g(0,0)=0$; the function $f$ is defined as $f(t, u):[0,1] \times[0, \infty) \rightarrow[0, \infty)$ and $\sigma(t), \tau(t)$ are continuous on $t$ and $0 \leq \sigma(t), \tau(t) \leq t$.

By using Banach contraction mapping principle and Leray-Schauder continuation principle, we obtain some sufficient conditions for the existence and uniqueness of the positive solutions for boundary value problem (4). Furthermore, we give an example to illustrate our results.

\section{Preliminary}

In this section, we introduce some definitions and preliminary facts which are used in this paper.

Definition 1 (see $[8,16])$. The fractional integral of order $\alpha$ with the lower limit $t_{0}$ for a function $f$ is defined as

$$
I^{\alpha}(f(t))=\frac{1}{\Gamma(\alpha)} \int_{t_{0}}^{t} \frac{f(s)}{(t-s)^{1-\alpha}} d s, \quad t>t_{0}, \alpha>0,
$$

provided the right-side is point-wise defined on $\left[t_{0}, \infty\right]$, where $\Gamma$ is the Gamma function.

Definition 2 (see $[8,16]$ ). Riemann-Liouville derivative of order $\alpha$ with the lower limit $t_{0}$ for a function $f:[0, \infty) \rightarrow \mathscr{R}$ can be written as

$$
\begin{array}{r}
D^{\alpha}(f(t))=\frac{1}{\Gamma(n-\alpha)} \frac{d^{n}}{d t^{n}} \int_{t_{0}}^{t} \frac{f(s)}{(t-s)^{\alpha+1-n}} d s, \\
t>t_{0}, n-1<\alpha<n .
\end{array}
$$

Definition 3 (see $[8,16]$ ). Caputo's derivative of order $\alpha$ with the lower limit $t_{0}$ for a function $f:[0, \infty) \rightarrow \mathscr{R}$ can be written as

$$
\begin{aligned}
{ }^{c} D^{\alpha}(f(t)) & =\frac{1}{\Gamma(n-\alpha)} \int_{t_{0}}^{t} \frac{f^{(n)}(s)}{(t-s)^{\alpha+1-n}} d s \\
& =I^{n-\alpha} f^{(n)}(t), \quad t>t_{0}, n-1<\alpha<n .
\end{aligned}
$$

It is well known that if $n-1 \leq \alpha \leq n$, then $D^{\alpha} t^{\alpha-k}=0$, $k=1,2, \ldots, n$. Furthermore, if $y(t) \in L^{1}[0, T]$ and $\alpha>0$, then for $t \in[0, T]$, we have

$$
D^{\alpha} I^{\alpha} y(t)=y(t)
$$

which is with the semigroup property

$$
I^{\delta} I^{\alpha}=I^{\delta+\alpha}=I^{\alpha} I^{\delta}
$$

for $\delta+\alpha>0$ and $t \in[0, T]$.

We also need to introduce some Lemmas as follows, which will be used in the proof of our main theorems.

Lemma 4 (see $[19,20]$ ). Let $\alpha>0$; then the fractional equation

$$
D^{\alpha}(h(t))=0 \text {, }
$$

has solutions

$$
\begin{aligned}
& h(t)=c_{1} t^{\alpha-1}+c_{2} t^{\alpha-2}+\cdots+c_{n} t^{\alpha-n}, \\
& c_{i} \in R, \quad i=1,2, \ldots, n, \quad n=[\alpha]+1 .
\end{aligned}
$$

Lemma 5 (see $[19,20])$. Let $\alpha>0$; then

$$
I^{\alpha} D^{\alpha} h(t)=h(t)+c_{1} t^{\alpha-1}+c_{2} t^{\alpha-2}+\cdots+c_{n} t^{\alpha-n}
$$

for some $c_{i} \in \mathscr{R}, i=1,2, \ldots, n, n=[\alpha]+1$.

Lemma 6 (see [28], the Banach contraction mapping theorem). Let $T: M \rightarrow M$ be a contraction mapping of a complete metric space $M$. Then $T$ has one and only one fixed point.

Lemma 7 (see [28-30], the Leray-Schauder continuation principle). Let $X$ be a Banach space with $C \subset X$ being closed and convex. Assume that $U$ is a relatively open subset of $C$ with $0 \in U$ and $T: \bar{U} \rightarrow C$ is completely continuous. Then either

(a) Thas a fixed point in $\bar{U}$, or

(b) there exists $u \in \partial U$ and $\lambda \in(0,1)$ with $u=\lambda T u$.

Throughout this paper, we assume that $f, g \in C([0,1] \times$ $[0, \infty),[0, \infty)$,$) and we satisfy the following$

(H): (i) $f(t, u), g(t, u)$ is Lebesgue measurable with respect to $t$ on $[0,1]$

(ii) $f(t, u), g(t, u)$ is continuous with respect to $u$ on $[0, \infty)$.

\section{Main Results}

For convenience, we rewrite (4) as follows:

$$
\begin{gathered}
D_{0+}^{\alpha} y(t)=p D_{0+}^{\beta} g(t, y(\sigma(t)))-f(t, y(\tau(t))), \\
0<t<1, \\
y(0)=0, \\
\eta y(\sigma(1))=y(1) .
\end{gathered}
$$


Abstract and Applied Analysis

3

Integrating both sides of (13) of $\alpha$ order with respect to $t$, it follows that

$$
\begin{aligned}
y(t) & \\
= & p \frac{1}{\Gamma(\alpha-\beta)} \int_{0}^{t}(t-s)^{\alpha-\beta-1} g(s, y(\sigma(s))) d s \\
& +c_{0} t^{\alpha-1}+c_{1} t^{\alpha-2}-\frac{1}{\Gamma(\alpha)} \\
& \cdot \int_{0}^{t}(t-s)^{\alpha-1} f(s, y(\tau(s))) d s, \quad 0<t<1 .
\end{aligned}
$$

From (14) and (15), we have

$$
c_{1}=0 \text {, }
$$

$y(1)$

$$
\begin{array}{r}
=p \frac{1}{\Gamma(\alpha-\beta)} \int_{0}^{1}(1-s)^{\alpha-\beta-1} g(s, y(\sigma(s))) d s \\
+c_{0}-\frac{1}{\Gamma(\alpha)} \int_{0}^{1}(1-s)^{\alpha-1} f(s, y(\tau(s))) d s, \\
0<t<1,
\end{array}
$$

$$
\begin{aligned}
& y(\sigma(1)) \\
& =p \frac{1}{\Gamma(\alpha-\beta)} \int_{0}^{\sigma(1)}[\sigma(1)-s]^{\alpha-\beta-1} g(s, y(\sigma(s))) d s \\
& \quad+c_{0} \sigma^{\alpha-1}(1)-\frac{1}{\Gamma(\alpha)} \\
& \quad \cdot \int_{0}^{\sigma(1)}[\sigma(1)-s]^{\alpha-1} f(s, y(\tau(s))) d s, \\
& \quad 0<t<1 .
\end{aligned}
$$

Combining (14) with (17), we obtain

$$
\begin{aligned}
c_{0}= & \frac{1}{\Gamma(\alpha)\left[1-\eta \sigma^{\alpha-1}(1)\right]} \\
& \cdot \int_{0}^{1}(1-s)^{\alpha-1} f(s, y(\tau(s))) d s \\
& -\frac{\eta}{\Gamma(\alpha)\left[1-\eta \sigma^{\alpha-1}(1)\right]} \\
& \cdot \int_{0}^{\sigma(1)}[\sigma(1)-s]^{\alpha-1} f(s, y(\tau(s))) d s \\
& -\frac{p}{\Gamma(\alpha-\beta)\left[1-\eta \sigma^{\alpha-1}(1)\right]} \\
& \cdot \int_{0}^{1}(1-s)^{\alpha-\beta-1} g(s, y(\sigma(s))) d s \\
& +\frac{p \eta}{\Gamma(\alpha-\beta)\left[1-\eta \sigma^{\alpha-1}(1)\right]}
\end{aligned}
$$

$$
\begin{array}{r}
\cdot \int_{0}^{\sigma(1)}[\sigma(1)-s]^{\alpha-\beta-1} g(s, y(\sigma(s))) d s \\
0<t<1 .
\end{array}
$$

According to (15) and (18), it follows that

$$
\begin{aligned}
& y(t) \\
& =\frac{p}{\Gamma(\alpha-\beta)} \int_{0}^{t}(t-s)^{\alpha-\beta-1} g(s, y(\sigma(s))) d s \\
& +\frac{1}{\Gamma(\alpha)\left[1-\eta \sigma^{\alpha-1}(1)\right]} \\
& \cdot \int_{0}^{1}(1-s)^{\alpha-1} t^{\alpha-1} f(s, y(\tau(s))) d s \\
& -\frac{\eta}{\Gamma(\alpha)\left[1-\eta \sigma^{\alpha-1}(1)\right]} \\
& \int_{0}^{\sigma(1)}[\sigma(1)-s]^{\alpha-1} t^{\alpha-1} f(s, y(\tau(s))) d s \\
& -\frac{p}{\Gamma(\alpha-\beta)\left[1-\eta \sigma^{\alpha-1}(1)\right]} \\
& \cdot \int_{0}^{1}(1-s)^{\alpha-\beta-1} t^{\alpha-1} g(s, y(\sigma(s))) d s \\
& +\frac{p \eta}{\Gamma(\alpha-\beta)\left[1-\eta \sigma^{\alpha-1}(1)\right]} \\
& \cdot \int_{0}^{\sigma(1)}[\sigma(1)-s]^{\alpha-\beta-1} t^{\alpha-1} g(s, y(\sigma(s))) d s \\
& -\frac{1}{\Gamma(\alpha)} \int_{0}^{t}(t-s)^{\alpha-1} f(s, y(\tau(s))) d s \\
& =\frac{p}{\Gamma(\alpha-\beta)} \int_{0}^{t}(t-s)^{\alpha-\beta-1} g(s, y(\sigma(s))) d s \\
& -\frac{p}{\Gamma(\alpha-\beta)\left[1-\eta \sigma^{\alpha-1}(1)\right]} \\
& \cdot\left(\int_{0}^{t}+\int_{t}^{\sigma(1)}+\int_{\sigma(1)}^{1}\right)(1-s)^{\alpha-\beta-1} t^{\alpha-1} g(s, y(\sigma(s))) d s \\
& -\frac{1}{\Gamma(\alpha)} \int_{0}^{t}(t-s)^{\alpha-1} f(s, y(\tau(s))) d s \\
& +\frac{1}{\Gamma(\alpha)\left[1-\eta \sigma^{\alpha-1}(1)\right]} \\
& \cdot\left(\int_{0}^{t}+\int_{0}^{\sigma(1)}+\int_{\sigma(1)}^{1}\right)(1-s)^{\alpha-1} t^{\alpha-1} f(s, y(\tau(s))) d s
\end{aligned}
$$




$$
\begin{aligned}
& +\frac{p \eta}{\Gamma(\alpha-\beta)\left[1-\eta \sigma^{\alpha-1}(1)\right]} \\
& \cdot\left(\int_{0}^{t}+\int_{t}^{\sigma(1)}\right)[\sigma(1)-s]^{\alpha-\beta-1} t^{\alpha-1} g(s, y(\sigma(s))) d s \\
& -\frac{\eta}{\Gamma(\alpha)\left[1-\eta \sigma^{\alpha-1}(1)\right]} \\
& \cdot\left(\int_{0}^{t}+\int_{t}^{\sigma(1)}\right)[\sigma(1)-s]^{\alpha-1} t^{\alpha-1} f(s, y(\tau(s))) d s \\
& =p \int_{0}^{t} \frac{1}{\Gamma(\alpha-\beta)\left[1-\eta \sigma^{\alpha-1}(1)\right]} \\
& \cdot\left\{\left[1-\eta \sigma^{\alpha-1}(1)\right](t-s)^{\alpha-\beta-1}\right. \\
& -(1-s)^{\alpha-\beta-1} t^{\alpha-1} \\
& +\eta[\sigma(1)-s]^{\alpha-\beta-1} t^{\alpha-1} \\
& \cdot g(s, y(\sigma(s)))\} d s \\
& +p \int_{t}^{\sigma(1)}\left(\left(-(1-s)^{\alpha-\beta-1} t^{\alpha-1}\right.\right. \\
& \left.+\eta[\sigma(1)-s]^{\alpha-\beta-1} t^{\alpha-1}\right) \\
& \left.\cdot\left(\Gamma(\alpha-\beta)\left[1-\eta \sigma^{\alpha-1}(1)\right]\right)^{-1}\right) \\
& \cdot g(s, y(\sigma(s))) d s \\
& -p \int_{\sigma(1)}^{1} \frac{(1-s)^{\alpha-\beta-1} t^{\alpha-1}}{\Gamma(\alpha-\beta)\left[1-\eta \sigma^{\alpha-1}(1)\right]} \\
& \cdot g(s, y(\sigma(s))) d s \\
& -\int_{0}^{t}\left(\left(\left(1-\eta \sigma^{\alpha-1}(1)\right)(t-s)^{\alpha-1}\right.\right. \\
& \left.-(1-s)^{\alpha-1} t^{\alpha-1}+\eta[\sigma(1)-s]^{\alpha-1} t^{\alpha-1}\right) \\
& \left.\cdot\left(\Gamma(\alpha)\left[1-\eta \sigma^{\alpha-1}(1)\right]\right)^{-1}\right) \\
& f(s, y(\tau(s))) d s \\
& -\int_{t}^{\sigma(1)} \frac{-(1-s)^{\alpha-1} t^{\alpha-1}+\eta[\sigma(1)-s]^{\alpha-1} t^{\alpha-1}}{\Gamma(\alpha)\left[1-\eta \sigma^{\alpha-1}(1)\right]} \\
& \text { - } f(s, y(\tau(s))) d s \\
& +\int_{\sigma(1)}^{1} \frac{(1-s)^{\alpha-1} t^{\alpha-1}}{\Gamma(\alpha)\left[1-\eta \sigma^{\alpha-1}(1)\right]} f(s, y(\tau(s))) d s,
\end{aligned}
$$$$
0<t<1
$$

Let

$G(t, s)$

$$
\left\{\begin{array}{c}
\left((1-s)^{\alpha-\beta-1} t^{\alpha-1}-\left(1-\eta \sigma^{\alpha-1}(1)\right)\right. \\
\left.\cdot(t-s)^{\alpha-\beta-1}-\eta[\sigma(1)-s]^{\alpha-\beta-1} t^{\alpha-1}\right) \\
\cdot\left(\Gamma(\alpha-\beta)\left[1-\eta \sigma^{\alpha-1}(1)\right]\right)^{-1} \\
0 \leq s \leq t \leq 1, \quad s \leq \sigma(1) \\
\left((1-s)^{\alpha-\beta-1} t^{\alpha-1}\right. \\
\left.-\left(1-\eta \sigma^{\alpha-1}(1)\right)(t-s)^{\alpha-\beta-1}\right) \\
\cdot\left(\Gamma(\alpha-\beta)\left[1-\eta \sigma^{\alpha-1}(1)\right]\right)^{-1} \\
0<\sigma(1) \leq s \leq t \leq 1 \\
\left((1-s)^{\alpha-\beta-1} t^{\alpha-1}-\eta[\sigma(1)-s]^{\alpha-\beta-1} t^{\alpha-1}\right) \\
\cdot\left(\Gamma(\alpha-\beta)\left[1-\eta \sigma^{\alpha-1}(1)\right]\right)^{-1} \\
0<t \leq s \leq \sigma(1) \leq 1 \\
\left((1-s)^{\alpha-\beta-1} t^{\alpha-1}\right) \\
\cdot\left(\Gamma(\alpha-\beta)\left[1-\eta \sigma^{\alpha-1}(1)\right]\right)^{-1} \\
0 \leq t \leq s \leq 1, \quad \sigma(1) \leq s
\end{array}\right.
$$

$\widetilde{G}(t, s)$

$$
\left\{\begin{array}{c}
\left((1-s)^{\alpha-1} t^{\alpha-1}-\left(1-\eta \sigma^{\alpha-1}(1)\right)(t-s)^{\alpha-1}\right. \\
\left.-\eta[\sigma(1)-s]^{\alpha-1} t^{\alpha-1}\right) \\
\cdot\left(\Gamma(\alpha)\left[1-\eta \sigma^{\alpha-1}(1)\right]\right)^{-1}, \\
0 \leq s \leq t \leq 1, \quad s \leq \sigma(1) \\
\left((1-s)^{\alpha-1} t^{\alpha-1}\right. \\
\left.-\left(1-\eta \sigma^{\alpha-1}(1)\right)(t-s)^{\alpha-1}\right) \\
\cdot\left(\Gamma(\alpha)\left[1-\eta \sigma^{\alpha-1}(1)\right]\right)^{-1} \\
0<\sigma(1) \leq s \leq t \leq 1 \\
\left((1-s)^{\alpha-1} t^{\alpha-1}-\eta[\sigma(1)-s]^{\alpha-1} t^{\alpha-1}\right) \\
\cdot\left(\Gamma(\alpha)\left[1-\eta \sigma^{\alpha-1}(1)\right]\right)^{-1} \\
0<t \leq s \leq \sigma(1) \leq 1 \\
\left((1-s)^{\alpha-1} t^{\alpha-1}\right) \\
\cdot\left(\Gamma(\alpha)\left[1-\eta \sigma^{\alpha-1}(1)\right]\right)^{-1} \\
0 \leq t \leq s \leq 1, \quad \sigma(1) \leq s .
\end{array}\right.
$$

According to (19)-(21), it follows that

$$
\begin{aligned}
y(t)= & -p \int_{0}^{1} G(t, s) g(s, y(\sigma(s))) d s \\
& +\int_{0}^{1} \widetilde{G}(t, s) f(s, y(\tau(s))) d s,
\end{aligned}
$$

which means that if $y(t)$ satisfies (13)-(14), then it satisfies (22). It is easy to show that if $y(t)$ satisfies (22), then it also satisfies (13)-(14). Thus, the boundary value problem (13)-(14) is actually equivalent to integral equation (22). Therefore, we have the following.

Lemma 8. Problem (13)-(14) is equivalent to (22).

Lemma 9. For any $(t, s) \in(0,1) \times(0,1), G(t, s), \widetilde{G}(t, s)$ are continuous and $G(t, s)>0, \widetilde{G}(t, s)>0$. 
Proof. It is obvious that $G(t, s), \widetilde{G}(t, s)$ are continuous on $[0,1] \times[0,1]$. We first prove that $G(t, s)>0$ on $[0,1] \times[0,1]$. Let

$$
\begin{aligned}
& g_{1}(t, s) \\
& =(1-s)^{\alpha-\beta-1} t^{\alpha-1}-\left[1-\eta \sigma^{\alpha-1}(1)\right](t-s)^{\alpha-\beta-1} \\
& \quad-\eta[\sigma(1)-s]^{\alpha-\beta-1} t^{\alpha-1}, \\
& \quad 0 \leq s \leq t \leq 1, \quad s \leq \sigma(1) ; \\
& g_{2}(t, s) \quad 0<\sigma(1) \leq s \leq t \leq 1 ; \\
& =(1-s)^{\alpha-\beta-1} t^{\alpha-1}-\left[1-\eta \sigma^{\alpha-1}(1)\right](t-s)^{\alpha-\beta-1}, \\
& g_{3}(t, s) \quad 0<t \leq s \leq \sigma(1) \leq 1 ; \\
& =(1-s)^{\alpha-\beta-1} t^{\alpha-1}-\eta[\sigma(1)-s]^{\alpha-\beta-1} t^{\alpha-1}, \\
& g_{4}(t, s)=(1-s)^{\alpha-\beta-1} t^{\alpha-1}, \quad 0 \leq t \leq s \leq 1, \sigma(1) \leq s .
\end{aligned}
$$

We first show that $g_{1}(t, s)>0,0 \leq s \leq t \leq 1, s \leq \sigma(1)$. We rewrite $g_{1}(t, s)$ as follows:

$$
\begin{aligned}
& g_{1}(t, s) \\
& =t^{\alpha-1}\left((1-s)^{\alpha-\beta-1}-\left[1-\eta \sigma^{\alpha-1}(1)\right]\right. \\
& \left.\quad\left(1-\frac{s}{t}\right)^{\alpha-\beta-1} t^{-\beta}-\eta[\sigma(1)-s]^{\alpha-\beta-1}\right) .
\end{aligned}
$$

Let

$$
\begin{aligned}
h_{1}(t, s)= & (1-s)^{\alpha-\beta-1} \\
& -\left[1-\eta \sigma^{\alpha-1}(1)\right]\left(1-\frac{s}{t}\right)^{\alpha-\beta-1} t^{-\beta} \\
& -\eta[\sigma(1)-s]^{\alpha-\beta-1} .
\end{aligned}
$$

Since $0<\eta \sigma^{\alpha-1}(1)<1$, then

$$
h_{1}(s, s)=(1-s)^{\alpha-\beta-1}-\eta[\sigma(1)-s]^{\alpha-\beta-1}>0 .
$$

Differentiating both sides of (25) with respect to $t$, it follows that

$$
\begin{aligned}
\frac{\partial h_{1}(t, s)}{\partial t} & \\
= & {\left[1-\eta \sigma^{\alpha-1}(1)\right]\left(1-\frac{s}{t}\right)^{\alpha-\beta-2} t^{-\beta-2} } \\
& \cdot\left[(\beta-\alpha+1) s+\left(1-\frac{s}{t}\right) t \beta\right] \\
= & {\left[1-\eta \sigma^{\alpha-1}(1)\right]\left(1-\frac{s}{t}\right)^{\alpha-\beta-2} t^{-\beta-2}(s-\alpha s+t \beta) \geq 0 }
\end{aligned}
$$

which means that $h_{1}(t, s)$ is nondecreasing with respect to $t$ on $[s, 1]$. Thus, for any $t \in[s, 1], h_{1}(t, s) \geq h_{1}(s, s)>0$, therefore, $g_{1}(t, s)=t^{\alpha-1} h_{1}(t, s)>0$.

Using the similar method, we can prove that $g_{2}(t, s)>0$, and it is obvious that $g_{3}(t, s)>0, g_{4}(t, s)>0$. Hence, combining (20) and (23), we obtain that $G(t, s)>0$.

Now, we prove that $\widetilde{G}(t, s)>0$. Denote

$$
\begin{aligned}
& \tilde{g}_{1}(t, s) \\
& =(1-s)^{\alpha-1} t^{\alpha-1}-\left[1-\eta \sigma^{\alpha-1}(1)\right](t-s)^{\alpha-1} \\
& -\eta[\sigma(1)-s]^{\alpha-1} t^{\alpha-1}, \\
& 0 \leq s \leq t \leq 1, \quad s \leq \sigma(1) ;
\end{aligned}
$$

$\widetilde{g}_{2}(t, s)$

$$
\begin{gathered}
=(1-s)^{\alpha-1} t^{\alpha-1}-\left[1-\eta \sigma^{\alpha-1}(1)\right](t-s)^{\alpha-1}, \\
0<\sigma(1) \leq s \leq t \leq 1 ; \\
\widetilde{g}_{3}(t, s) \\
=(1-s)^{\alpha-1} t^{\alpha-1}-\eta[\sigma(1)-s]^{\alpha-1} t^{\alpha-1}, \\
0<t \leq s \leq \sigma(1) \leq 1 ; \\
\widetilde{g}_{4}(t, s)=(1-s)^{\alpha-1} t^{\alpha-1}, \quad 0 \leq t \leq s \leq 1, \sigma(1) \leq s .
\end{gathered}
$$

Let

$$
\begin{aligned}
\tilde{h}_{1}(t, s)= & (1-s)^{\alpha-1}-\left[1-\eta \sigma^{\alpha-1}(1)\right]\left(1-\frac{s}{t}\right)^{\alpha-1} \\
& -\eta[\sigma(1)-s]^{\alpha-1} .
\end{aligned}
$$

Since $0<\eta \sigma^{\alpha-1}(1)<1$, then

$$
\begin{aligned}
\tilde{h}_{1}(1, s) \geq & (1-s)^{\alpha-1}-\left[1-\eta \sigma^{\alpha-1}(1)\right](1-s)^{\alpha-1} \\
& -\eta[\sigma(1)-s]^{\alpha-1} \\
= & \eta \sigma^{\alpha-1}(1)(1-s)^{\alpha-1}-\eta[\sigma(1)-s]^{\alpha-1} \\
= & \eta[\sigma(1)-s \sigma(1)]^{\alpha-1}-\eta[\sigma(1)-s]^{\alpha-1} \\
\geq & \eta[\sigma(1)-s]^{\alpha-1}-\eta[\sigma(1)-s]^{\alpha-1}=0 .
\end{aligned}
$$

Differentiating both sides of (29) with respect to $t$, it follows that

$$
\begin{aligned}
\frac{\partial \tilde{h}_{1}(t, s)}{\partial t} & =-(\alpha-1)\left[1-\eta \sigma^{\alpha-1}(1)\right]\left(1-\frac{s}{t}\right)^{\alpha-2} s t^{-2} \\
& \leq 0, \quad 0<s<t \leq 1
\end{aligned}
$$

which means that $\widetilde{h}_{1}(t, s)$ is nonincreasing with respect to $t$ on $[s, 1]$. Thus, for any $t \in[s, 1], \widetilde{h}_{1}(t, s) \geq h_{1}(1, s)>0$, therefore, $\widetilde{g}_{1}(t, s)=t^{\alpha-1} \widetilde{h}_{1}(t, s)>0$.

Using the similar method, we can prove that $\tilde{g}_{2}(t, s)>$ 0 , and the case that $\widetilde{g}_{3}(t, s)>0, \widetilde{g}_{4}(t, s)>0$ is obvious. Combining (21) and (28) and using the above argument, we obtain that $\widetilde{G}(t, s)>0$. The proof is complete. 
Lemma 10. For any $(t, s) \in(0,1) \times(0,1), G(t, s), \widetilde{G}(t, s)$ are nondecreasing functions with respect to $t \in(0,1)$; that is, for any $t \in(0,1), G(t, s)>G(s, s), \widetilde{G}(t, s)>\widetilde{G}(s, s)$.

Proof. According to the proof of Lemma 9, we notice that

$$
\begin{gathered}
\frac{\partial g_{1}(t, s)}{\partial t}=(\alpha-1) t^{\alpha-2} h_{1}(t, s)+t^{\alpha-1} \frac{\partial h_{1}(t, s)}{\partial t}>0, \\
\quad 0<s<t \leq 1, \\
\frac{\partial g_{2}(t, s)}{\partial t}>0, \\
\frac{\partial g_{3}(t, s)}{\partial t}>0, \\
\frac{\partial g_{4}(t, s)}{\partial t}>0 .
\end{gathered}
$$

At the same time, for $s<t<1$, we have

$$
\begin{gathered}
\frac{\partial \widetilde{g}_{1}(t, s)}{\partial t} \\
=(\alpha-1)(1-s)^{\alpha-1} t^{\alpha-2}-\left[1-\eta \sigma^{\alpha-1}(1)\right] \\
\cdot(t-s)^{\alpha-2}-\eta[\sigma(1)-s]^{\alpha-1} t^{\alpha-2} \\
\geq(\alpha-1) t^{\alpha-2}\left[(1-s)^{\alpha-1}-\eta[\sigma(1)-s]^{\alpha-1}\right] \geq 0, \\
\frac{\partial \widetilde{g}_{2}(t, s)}{\partial t}>0, \\
\frac{\partial \widetilde{g}_{3}(t, s)}{\partial t}>0, \\
\frac{\partial \widetilde{g}_{4}(t, s)}{\partial t}>0 .
\end{gathered}
$$

The proof is complete.

Now, we present our main results.

Theorem 11. Assume that $(H)$ holds. Suppose that there are two functions $\lambda(t), \mu(t) \in C([0,1],[0, \infty))$ such that

$$
\begin{gathered}
|f(t, u)-f(t, v)| \leq \lambda(t)|u-v|, \\
\text { for } t \in[0,1], u, v \in[0, \infty), \\
|g(t, u)-g(t, v)| \leq \mu(t)|u-v|, \\
\text { for } t \in[0,1], u, v \in[0, \infty) .
\end{gathered}
$$

If

$$
-p \int_{0}^{1} G(1, s) \mu(s) d s+\int_{0}^{1} \widetilde{G}(1, s) \lambda(s)<1,
$$

then the problem (13)-(14) has a unique positive solution.

Proof. Set

$$
\Omega=\{y(t) \in C[0,1] \mid y(t) \geq 0, \text { for } t \in[0,1]\},
$$

with the maximum norm

$$
\|y\|=\max _{0 \leq t \leq 1}|y(t)| .
$$

It is easy to show that $\Omega$ is a complete metric space. We denote a operator $T$ as follows:

$$
\begin{aligned}
T y(t)= & -p \int_{0}^{1} G(t, s) g(s, y(\sigma(s))) d s \\
& +\int_{0}^{1} \widetilde{G}(t, s) f(s, y(\tau(s))) d s .
\end{aligned}
$$

From Lemma 9 and the conditions $p \leq 0, f>0, g>0$, it follows that $T$ maps $\Omega$ into itself and we only need to prove the contraction. In fact, according $(\mathrm{H})$ and (34), for any $u, v \in \Omega$, we have

$$
\begin{aligned}
& \|T u-T v\| \\
& =\max _{0 \leq t \leq 1} \mid-p \int_{0}^{1} G(t, s) g(s, u(\sigma(s))) d s \\
& +p \int_{0}^{1} G(t, s) g(s, v(\sigma(s))) d s \\
& +\int_{0}^{1} \widetilde{G}(t, s) f(s, u(\tau(s))) d s \\
& -\int_{0}^{1} \widetilde{G}(t, s) f(s, v(\tau(s))) d s \\
& \leq-p \int_{0}^{1} G(t, s) \mu(s)|u(\sigma(s))-v(\sigma(s))| d s \\
& +\int_{0}^{1} \widetilde{G}(t, s) \lambda(s)|u(\tau(s))-v(\tau(s))| d s \\
& \leq\left[-p \int_{0}^{1} G(t, s) \mu(s) d s+\int_{0}^{1} \widetilde{G}(t, s) \lambda(s)\right]\|u-v\| \\
& \leq\left[-p \int_{0}^{1} G(1, s) \mu(s) d s+\int_{0}^{1} \widetilde{G}(1, s) \lambda(s)\right]\|u-v\| \\
& <\|u-v\|,
\end{aligned}
$$

which means that

$$
|T u-T v|<\|u-v\| .
$$

By the Banach contraction mapping principle (Lemma 6), we obtain that $T$ has a unique fixed point $\widetilde{y}(t)$ which is a positive solution of (13)-(14). The proof is complete.

Remark 12. If $p=0, f(t, y(\tau(t)))=f(y(t))$, then problem (13)-(14) is problem (3). 
Theorem 13. Assume that $(H)$ holds. Suppose that there exists four nonnegative real-valued functions $m, n, l, q \in L^{1}[0,1]$ such that

$$
f(t, u) \leq n(t)+m(t) u,
$$

for almost every $t \in[0,1]$ and all $u \in[0, \infty)$,

$$
g(t, u) \leq l(t)+q(t) u,
$$

for almost every $t \in[0,1]$ and all $u \in[0, \infty)$.

If

$$
\int_{0}^{1}[\widetilde{G}(1, s) m(s)-p G(1, s) q(s)] d s<1,
$$

then the problem (13)-(14) has at least one positive solution.

Proof. We also consider the operator $T$ defined in (38). We divide the proof into four steps.

Step 1. $T: \Omega \rightarrow \Omega$ is continuous.

Let $y_{n}(t)$ be a sequence in $\Omega$ such that $y_{n}(t) \rightarrow y(t)$ as $n \rightarrow \infty$. Noticing that $f(t, y), g(t, y)$ are continuous with respect to $y$, then for each $t \in[0,1]$, we have

$$
\begin{aligned}
\lim _{n \rightarrow \infty} f\left(t, y_{n}(\tau(t))\right) & =f(t, y(\tau(t))), \\
\lim _{n \rightarrow \infty}\left(t, y_{n}(\sigma(t))\right) & =g(t, y(\sigma(t))) ;
\end{aligned}
$$

thus,

$$
\begin{aligned}
& \lim _{n \rightarrow \infty} \sup _{t \in[0,1]}\left|f\left(t, y_{n}(\tau(t))\right)-f(t, y(\tau(t)))\right|=0, \\
& \lim _{n \rightarrow \infty} \sup _{t \in[0,1]}\left|g\left(t, y_{n}(\sigma(t))\right)-g(t, y(\sigma(t)))\right|=0,
\end{aligned}
$$

which implies that

$$
\begin{aligned}
& \left|T y_{n}(t)-T y(t)\right| \\
& =\mid-p \int_{0}^{1} G(t, s) g\left(s, y_{n}(\sigma(s))\right) d s \\
& \quad+p \int_{0}^{1} G(t, s) g(s, y(\sigma(s))) d s \\
& \quad+\int_{0}^{1} \widetilde{G}(t, s) f\left(s, y_{n}(\sigma(s))\right) d s \\
& \quad-\int_{0}^{1} \widetilde{G}(t, s) f(s, y(\sigma(s))) d s \mid
\end{aligned}
$$

$$
\begin{aligned}
\leq & -p \int_{0}^{1} G(t, s)\left|g\left(s, y_{n}(\sigma(s))\right)-g(s, y(\sigma(s)))\right| d s \\
& +\int_{0}^{1} \widetilde{G}(t, s)\left|f\left(s, y_{n}(\sigma(s))\right)-f(s, y(\sigma(s)))\right| d s \\
\leq & -p \int_{0}^{1} G(1, s) d s \sup _{t \in[0,1]}\left|g\left(s, y_{n}(\sigma(s))\right)-g(s, y(\sigma(s)))\right| \\
& +\int_{0}^{1} \widetilde{G}(1, s) d s \sup _{t \in[0,1]}\left|f\left(s, y_{n}(\sigma(s))\right)-f(s, y(\sigma(s)))\right| \\
\longrightarrow & 0, \quad \text { as } n \longrightarrow \infty .
\end{aligned}
$$

Step 2. T maps bounded sets into bounded sets in $\Omega$.

Indeed, it suffices to show that for any $\gamma>0$, there exists a positive constant $\eta^{\star}>0$ such that for $y \in B_{\gamma}=\{y \in \Omega$ : $\|y\| \leq \gamma\}$, we have $\|T y\| \leq \eta^{\star}$. From (38)-(43), we have

$$
\begin{aligned}
& \|T y\| \\
& =\max _{0 \leq t \leq 1} \mid-p \int_{0}^{1} G(t, s) g(s, y(\sigma(s))) d s \\
& +\int_{0}^{1} \widetilde{G}(t, s) f(s, y(\tau(s))) d s \\
& \leq-p \int_{0}^{1} G(t, s)[l(s)+q(s) y(\sigma(s))] d s \\
& +\int_{0}^{1} \widetilde{G}(t, s)[n(s)+m(s) y(\tau(s))] d s \\
& \leq \int_{0}^{1}[-p G(1, s) l(s)+\widetilde{G}(1, s) n(s)] d s \\
& +\int_{0}^{1}[-p G(1, s) q(s)+\widetilde{G}(1, s) m(s)] d s\|y\| \\
& \leq \int_{0}^{1}[-p G(1, s) l(s)+\widetilde{G}(1, s) n(s)] d s+\gamma:=\eta^{\star},
\end{aligned}
$$

which means

$$
\|T y\| \leq \eta^{\star} .
$$

Step 3. T maps bounded sets into equicontinuous sets in $\Omega$.

For any $t_{1}, t_{2} \in[0,1], t_{1}<t_{2}$, and for each $y(t) \in B_{\gamma}$, we have

$$
\begin{aligned}
& \left|T y\left(t_{1}\right)-T y\left(t_{2}\right)\right| \\
& =\mid-p \int_{0}^{1}\left[G\left(t_{1}, s\right)-G\left(t_{2}, s\right)\right] g(s, y(\sigma(s))) d s \\
& \quad+\int_{0}^{1}\left[\widetilde{G}\left(t_{1}, s\right)-\widetilde{G}\left(t_{2}, s\right)\right] f(s, y(\tau(s))) d s \mid
\end{aligned}
$$




$$
\begin{aligned}
\leq & -p \int_{0}^{1}\left|G\left(t_{1}, s\right)-G\left(t_{2}, s\right)\right|[l(s)+q(s) y(\sigma(s))] d s \\
& +\int_{0}^{1}\left|\widetilde{G}\left(t_{1}, s\right)-\widetilde{G}\left(t_{2}, s\right)\right|[n(t)+m(t) y(\tau(s))] d s \\
\leq & -p \int_{0}^{1}\left|G\left(t_{1}, s\right)-G\left(t_{2}, s\right)\right|[l(s)+q(s) \gamma] d s \\
& +\int_{0}^{1}\left|\widetilde{G}\left(t_{1}, s\right)-\widetilde{G}\left(t_{2}, s\right)\right|[n(t)+m(t) \gamma] d s .
\end{aligned}
$$

Because $G(t, s), \widetilde{G}(t, s)$ are continuous on $[0,1] \times[0,1]$, it is uniformly continuous on $[0,1] \times[0,1]$, which means that for any $\varepsilon>0$, there exists $\delta>0$, when $\left|t_{2}-t_{1}\right|<\delta, s \in[0,1]$,

$$
\begin{aligned}
& \left|G\left(t_{1}, s\right)-G\left(t_{2}, s\right)\right|<\frac{\varepsilon}{2(-p) \int_{0}^{1}[l(s)+q(s) \gamma] d s}, \\
& \left|\widetilde{G}\left(t_{1}, s\right)-\widetilde{G}\left(t_{2}, s\right)\right|<\frac{\varepsilon}{2 \int_{0}^{1}[n(s)+m(s) \gamma] d s} .
\end{aligned}
$$

Thus

$$
\left|T y\left(t_{1}\right)-T y\left(t_{2}\right)\right|<\varepsilon
$$

which means that $\left\{T y: y \in B_{\gamma}\right\}$ is equicontinuous.

Step 4. A priori bounds.

Let

$$
U=\{y \in \Omega:\|y\|<r\}
$$

$$
\text { where } r=\frac{\int_{0}^{1}[\widetilde{G}(1, s) n(s)-p G(1, s) l(s)] d s}{1-\int_{0}^{1}[\widetilde{G}(1, s) m(s)-p G(1, s) q(s)] d s} \text {. }
$$

Assume that there exists $y \in U$ and $0<\lambda<1$ such that $y=\lambda T y$ and we claim that $\|y\| \neq r$. In fact,

$$
\begin{aligned}
& y(t)= \lambda(T y)(t) \\
&=\lambda\left[-p \int_{0}^{1} G(t, s) g(s, y(\sigma(s))) d s\right. \\
& \\
&\left.\quad+\int_{0}^{1} \widetilde{G}(t, s) f(s, y(\tau(s))) d s\right],
\end{aligned}
$$

which implies that

$$
\begin{aligned}
& |y(t)| \\
& =\lambda \mid-p \int_{0}^{1} G(t, s) g(s, y(\sigma(s))) d s \\
& \quad+\int_{0}^{1} \widetilde{G}(t, s) f(s, y(\tau(s))) d s \mid \\
& <-p \int_{0}^{1} G(t, s)[l(s)+q(s) y(\sigma(s))] d s \\
& \quad+\int_{0}^{1} \widetilde{G}(t, s)[n(s)+m(s) y(\tau(s))] d s \\
& \leq \int_{0}^{1}[-p G(1, s) l(s)+\widetilde{G}(1, s) n(s)] d s \\
& \quad+\int_{0}^{1}[-p G(1, s) q(s)+\widetilde{G}(1, s) m(s)] d s\|y\| \\
& \leq \int_{0}^{1}[-p G(1, s) l(s)+\widetilde{G}(1, s) n(s)] d s \\
& \quad+r \int_{0}^{1}[-p G(1, s) q(s)+\widetilde{G}(1, s) m(s)] d s=r
\end{aligned}
$$

which means that

$$
\|y\|<r .
$$

That is, there is no $y \in \partial U$, such that $y=\lambda T y$ for $0<\lambda<1$.

From Step 1 to Step 3, it follows that $T$ is completely continuous. Along with Step 4 and Lemma 7, it follows that $T$ has at least a fixed point in $U$. The proof is complete.

\section{Examples}

Example 1. Consider

$$
\begin{gathered}
D^{3 / 2} x(t)+\frac{1}{2} D^{3 / 4}\left(\frac{t^{2} x(t / 2)}{10[1+x(t / 2)]}\right) \\
=\frac{e^{-t} x(t / 2)}{\left(9+e^{t}\right)[1+x(t / 2)]}, \quad t \in[0,1], \\
x(0)=0, \\
x(1)=x\left(\frac{1}{2}\right),
\end{gathered}
$$


where

$$
\begin{aligned}
n & =2, \\
p & =-\frac{1}{2}, \\
\alpha & =\frac{3}{2} \\
\beta & =\frac{3}{4}, \\
\eta & =1, \\
\tau(t) & =\sigma(t)=\frac{t}{2}, \\
g & =\frac{t^{2} x(t / 2)}{10[1+x(t / 2)]}, \\
f & =\frac{e^{-t} x(t / 2)}{\left(9+e^{t}\right)[1+x(t / 2)]} .
\end{aligned}
$$

It is easy to show that

$$
\begin{aligned}
& \lambda(t)=\frac{e^{-t}}{9+e^{t}}, \\
& \mu(t)=\frac{t^{2}}{10} .
\end{aligned}
$$

Let

$$
h(x)=\frac{x}{1+x}, \quad x>0
$$

then

$$
\begin{aligned}
|h(x)-h(y)| & =\left|\frac{x}{1+x}-\frac{y}{1+y}\right|=\frac{|x-y|}{(1+x)(1+y)} \\
& <|x-y|, \quad \text { for } x, y>0 ;
\end{aligned}
$$

thus,

$$
\begin{aligned}
& |f(t, u)-f(t, v)| \leq \lambda(t)|u-v|, \\
& |g(t, u)-g(t, v)| \leq \mu(t)|u-v|,
\end{aligned}
$$

which satisfies (28), (29). Because

$$
G(1, s)=\left\{\begin{array}{c}
\left((1-s)^{-1 / 4}-(1-1 / \sqrt{2})\right. \\
\left.\cdot(1-s)^{-1 / 4}-(1 / 2-s)^{-1 / 4}\right) \\
\cdot(\Gamma(3 / 4)(1-1 / \sqrt{2}))^{-1}, \\
0 \leq s \leq \frac{1}{2} ; \\
\left((1-s)^{-1 / 4}-(1-1 / \sqrt{2})(1-s)^{-1 / 4}\right) \\
\cdot(\Gamma(3 / 4)(1-1 / \sqrt{2}))^{-1}, \\
\widetilde{G}(1, s)=\left\{\begin{array}{c}
\left((1-s)^{1 / 2}-(1-1 / \sqrt{2})\right. \\
\left.\cdot(1-s)^{1 / 2}-(1 / 2-s)^{1 / 2}\right) \\
\cdot(\Gamma(3 / 2)(1-1 / \sqrt{2}))^{-1}, \\
0 \leq s \leq \frac{1}{2} ; \\
\left((1-s)^{1 / 2}-(1-1 / \sqrt{2})(1-s)^{1 / 2}\right) \\
\cdot(\Gamma(3 / 2)(1-1 / \sqrt{2}))^{-1},
\end{array}\right.
\end{array}\right.
$$

thus

$$
\begin{aligned}
-p & \int_{0}^{1} G(1, s) \mu(s) d s+\int_{0}^{1} \widetilde{G}(1, s) \lambda(s) \\
= & \frac{1}{2} \int_{0}^{1 / 2} \frac{1 / \sqrt{2}(1-s)^{-1 / 4}-(1 / 2-s)^{-1 / 4}}{\Gamma(3 / 4)(1-1 / \sqrt{2})} \frac{s^{2}}{10} d s \\
& +\frac{1}{2} \int_{1 / 2}^{1} \frac{1 / \sqrt{2}(1-s)^{-1 / 4}}{\Gamma(3 / 4)(1-1 / \sqrt{2})} \frac{s^{2}}{10} d s \\
& +\int_{0}^{1 / 2} \frac{1 / \sqrt{2}(1-s)^{1 / 2}-(1 / 2-s)^{1 / 2}}{\Gamma(3 / 2)(1-1 / \sqrt{2})} \frac{e^{-s}}{9+e^{s}} d s \\
& +\int_{1 / 2}^{1} \frac{1 / \sqrt{2}(1-s)^{1 / 2}}{\Gamma(3 / 2)(1-1 / \sqrt{2})} \frac{e^{-s}}{9+e^{s}} d s \\
< & \frac{1}{2} \int_{0}^{1 / 2} \frac{(1-s)^{-1 / 4}}{\Gamma(3 / 4)(\sqrt{2}-1)} \frac{s^{2}}{10} d s \\
& +\frac{1}{2} \int_{1 / 2}^{1} \frac{(1-s)^{-1 / 4}}{\Gamma(3 / 4)(\sqrt{2}-1)} \frac{s^{2}}{10} d s
\end{aligned}
$$$$
+\int_{0}^{1 / 2} \frac{(1-s)^{1 / 2}}{\Gamma(3 / 2)(\sqrt{2}-1)} \frac{e^{-s}}{9+e^{s}} d s
$$ 


$$
\begin{aligned}
& <\frac{1}{20 \Gamma(3 / 4)(\sqrt{2}-1)}+\frac{1}{9 \Gamma(3 / 2)(\sqrt{2}-1)} \\
& \approx 0.4014<1 ;
\end{aligned}
$$

which satisfies Theorem 11. Thus (56) has a unique positive solution on $[0,1]$.

\section{Conflict of Interests}

The authors declare that they have no competing interests.

\section{Acknowledgments}

The work was supported by NSF of Hunan Province (no. 13JJ3074), the Project of Department of Education of Hunan Province (13A088 and 12C0361), the Scientific Research Foundation of Hengyang City (nos. J1, 2012KJ2, and 2012KJ3) and the construct program in USC.

\section{References}

[1] V. Lakshmikantham, "Theory of fractional functional differential equations," Nonlinear Analysis: Theory, Methods and Applications, vol. 69, no. 10, pp. 3337-3343, 2008.

[2] V. Lakshmikantham and A. S. Vatsala, "Basic theory of fractional differential equations," Nonlinear Analysis: Theory, Methods 'Applications, vol. 69, no. 8, pp. 2677-2682, 2008.

[3] Y. Zhou, F. Jiao, and J. Li, "Existence and uniqueness for fractional neutral differential equations with infinite delay," Nonlinear Analysis: Theory, Methods and Applications, vol. 71, no. 7-8, pp. 3249-3256, 2009.

[4] V. Lakshmikantham, S. Leela, and J. V. Devi, Theory of fractional Dynamic Systems, Cambridge Scientiffic, Cambridge, UK, 2009.

[5] N. Kosmatov, "Integral equations and initial value problems for nonlinear differential equations of fractional order," Nonlinear Analysis: Theory, Methods and Applications, vol. 70, no. 7, pp. 2521-2529, 2009.

[6] D. Araya and C. Lizama, "Almost automorphic mild solutions to fractional differential equations," Nonlinear Analysis, vol. 69, no. 11, pp. 3692-3705, 2008.

[7] R. P. Agarwal, Y. Zhou, and Y. He, "Existence of fractional neutral functional differential equations," Computers and Mathematics with Applications, vol. 59, no. 3, pp. 1095-1100, 2010.

[8] A. A. Kilbas, H. M. Srivastava, and J. J. Trujillo, Theory and Applications of Fractional Differential Equations, vol. 204 of North Holland Mathematics Studies, Elsevier, Amsterdam, The Netherlands, 2006.

[9] Z. Bai, "On positive solutions of a nonlocal fractional boundary value problem," Nonlinear Analysis: Theory, Methods and Applications, vol. 72, no. 2, pp. 916-924, 2010.

[10] Z. Odibat and S. Momani, "A generalized differential transform method for linear partial differential equations of fractional order," Applied Mathematics Letters, vol. 21, no. 2, pp. 194-199, 2008.

[11] S. Momani and Z. Odibat, "A novel method for nonlinear fractional partial differential equations: combination of DTM and generalized Taylor's formula," Journal of Computational and Applied Mathematics, vol. 220, no. 1-2, pp. 85-95, 2008.
[12] M. M. Meerschaert and C. Tadjeran, "Finite difference approximations for two-sided space-fractional partial differential equations," Applied Numerical Mathematics, vol. 56, no. 1, pp. 80-90, 2006.

[13] S. Momani, Z. Odibat, and V. S. Erturk, "Generalized differential transform method for solving a space- and time-fractional diffusion-wave equation," Physics Letters A, vol. 370, no. 5-6, pp. 379-387, 2007.

[14] M. M. El-Borai, "Exact solutions for some nonlinear fractional parabolic partial differential equations," Applied Mathematics and Computation, vol. 206, no. 1, pp. 150-153, 2008.

[15] J. L. Wu, "A wavelet operational method for solving fractional partial differential equations numerically," Applied Mathematics and Computation, vol. 214, no. 1, pp. 31-40, 2009.

[16] I. Podlubny, Fractional Differential Equations, vol. 198 of Mathematics in Science and Engineering, Academic Press, San Diego, Calif, USA, 1993.

[17] M. El-Shahed, "Positive solutions for boundary value problem of nonlinear fractional differential equation," Abstract and Applied Analysis, vol. 2007, Article ID 10368, 8 pages, 2007.

[18] E. R. Kaufmann and E. Mboumi, "Positive solutions of a boundary value problem for a nonlocal fractional differential equations," The Electronic Journal of Qualitative Theory of Differential Equations, vol. 3, pp. 1-11, 2008.

[19] S. Liang and J. Zhang, "Positive solutions for boundary value problems of nonlinear fractional differential equation," Nonlinear Analysis: Theory, Methods and Applications, vol. 71, no. 11, pp. 5545-5550, 2009.

[20] Z. Bai and H. Lü, "Positive solutions for boundary value problem of nonlinear fractional differential equation," Journal of Mathematical Analysis and Applications, vol. 311, no. 2, pp. 495-505, 2005.

[21] A. Babakhani and V. Daftardar-Gejji, "Existence of positive solutions of nonlinear fractional differential equations," Journal of Mathematical Analysis and Applications, vol. 278, no. 2, pp. 434-442, 2003.

[22] C. Bai and J. Fang, "The existence of a positive solution for a singular coupled system of nonlinear fractional differential equations," Applied Mathematics and Computation, vol. 150, no. 3, pp. 611-621, 2004.

[23] M. Benchohra, S. Hamani, and S. K. Ntouyas, "Boundary value problems for differential equations with fractional order and nonlocal conditions," Nonlinear Analysis: Theory, Methods and Applications, vol. 71, no. 7-8, pp. 2391-2396, 2009.

[24] S. Q. Zhang, "Existence of positive solution for some class of nonlinear fractional differential equations," Journal of Mathematical Analysis and Applications, vol. 278, no. 1, pp. 136-148, 2003.

[25] C. Bai, "Positive solutions for nonlinear fractional differential equations with coefficient that changes sign," Nonlinear Analysis: Theory, Methods and Applications, vol. 64, no. 4, pp. 677-685, 2006.

[26] W. H. Deng, "Smoothness and stability of the solutions for nonlinear fractional differential equations," Nonlinear Analysis: Theory, Methods and Applications, vol. 72, no. 3-4, pp. 1768-1777, 2010.

[27] D. Delbosco, "Fractional calculus and function spaces," Journal of Fractional Calculus, vol. 6, pp. 45-53, 1994.

[28] R. P. Agarmal, M. Meehan, and D. O'Regan, Fixed Point Theory and Applications, Cambridge University Press, Cambridge, UK, 2001. 
[29] A. Granas, R. B. Guenther, and J. W. Lee, "Some general existence principles in the Caratheodory theory of nonlinear differential systems," Journal de Mathématiques Pures et Appliquées. Neuvième Serie, vol. 70, no. 2, pp. 153-196, 1991.

[30] C. F. Li, X. N. Luo, and Y. Zhou, "Existence of positive solutions of the boundary value problem for nonlinear fractional differential equations," Computers and Mathematics with Applications, vol. 59, no. 3, pp. 1363-1375, 2010. 


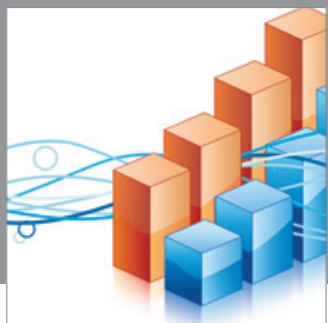

Advances in

Operations Research

mansans

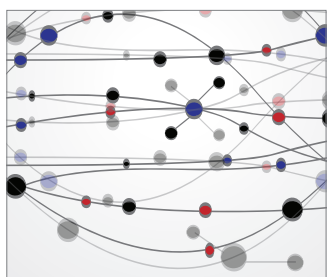

The Scientific World Journal
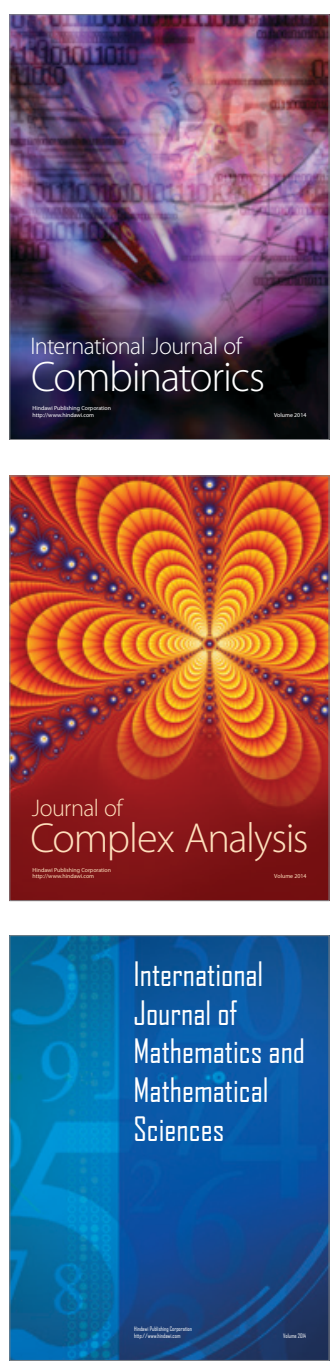
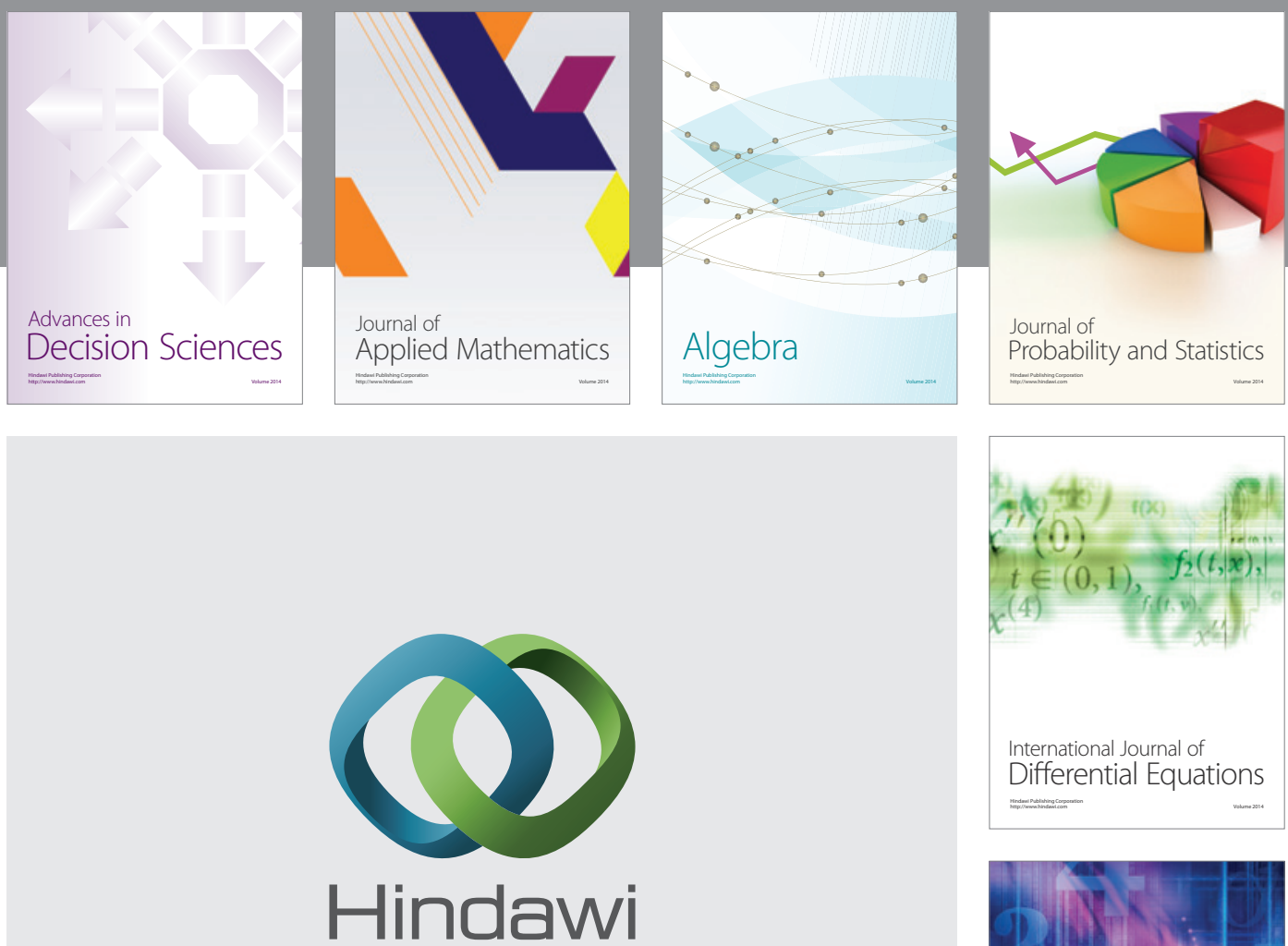

Submit your manuscripts at http://www.hindawi.com
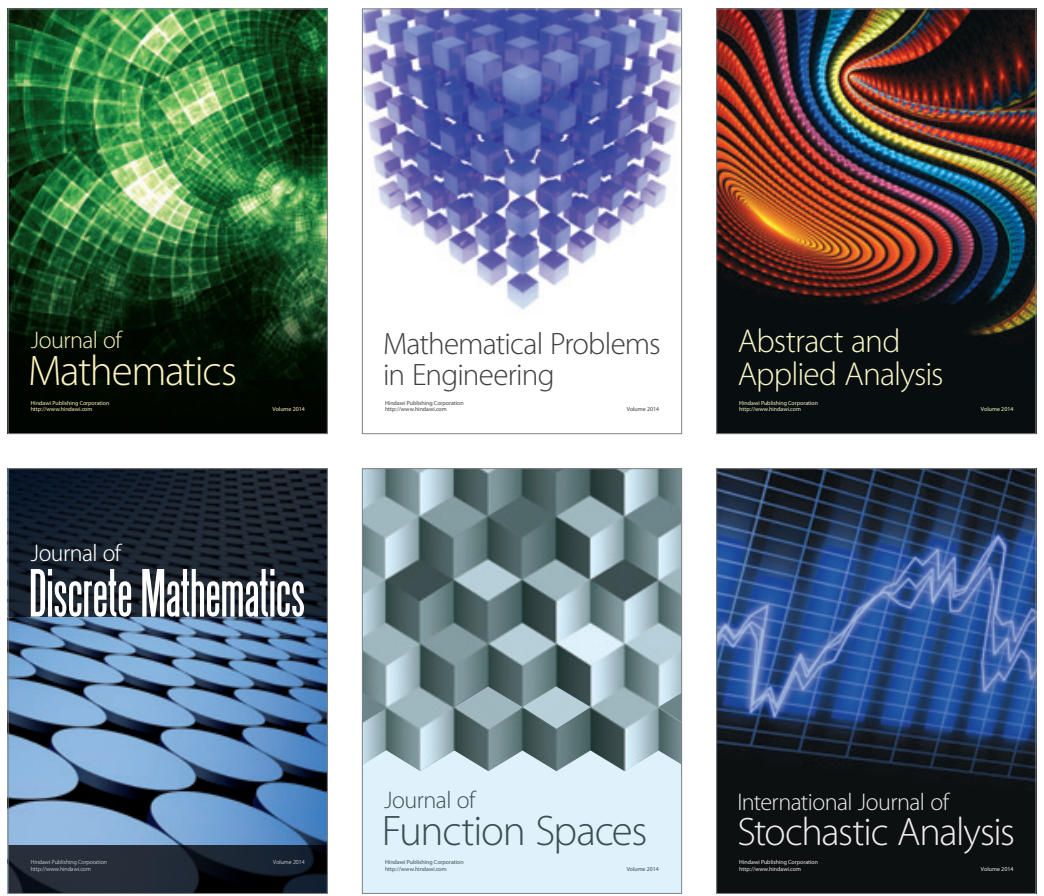

Journal of

Function Spaces

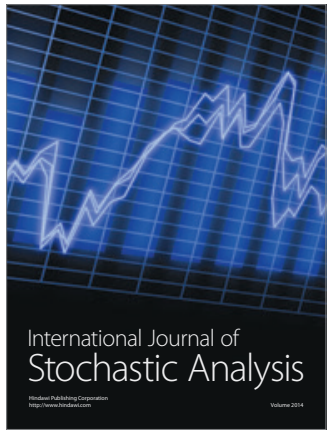

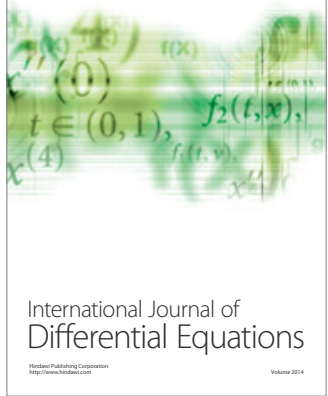
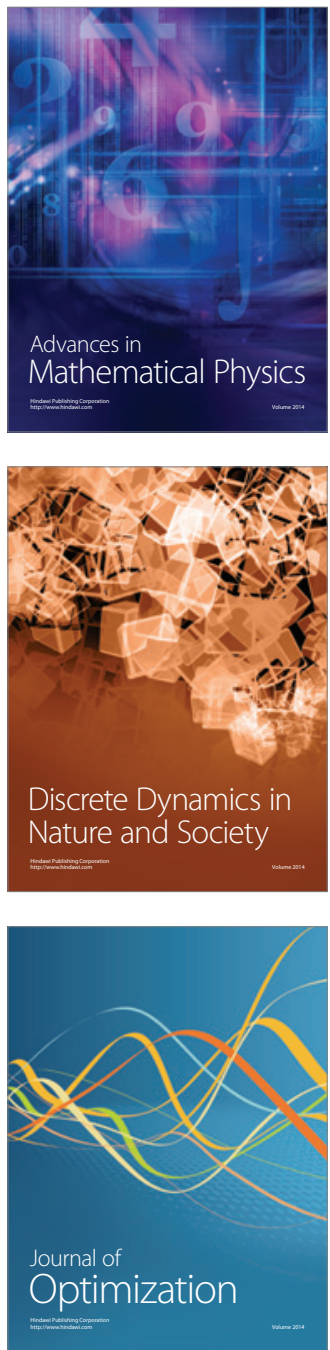